\title{
DESESCOLARIZAR LA VIDA. IVAN ILLICH Y LA CRÍTICA DE LAS INSTITUCIONES EDUCATIVAS,
}

por Jon IgELmo Zaldívar. Madrid: Enclave de libros, 2016, 223 páginas. ISBN: 97884-944529-I-8.

Este nuevo acercamiento a la obra de Iván Illich que propone el libro que aquí se presenta, tiene la finalidad expresa de «contribuir a la crítica de las certezas que fomentan la desesperanza de la gran mayoría de la población del siglo XXI» (p. 23). Es un propósito acorde con nuestro tiempo presente; el trabajo del profesor Igelmo aparece en un contexto marcado por incertidumbres diversas que exceden aquellas que el ser humano se ha planteado desde los albores de la humanidad. Nuevas dudas y vacilaciones han surgido a la sombra de la crisis del capitalismo financiero instalada en nuestras vidas desde hace varios años conmoviendo las renovadas certezas del crecimiento sin límites. Muy al contrario, lejos de ese dogma, esta crisis mostró el aumento de las desigualdades, la cada vez mayor distancia entre clases sociales, el significativo aumento de la pobreza, la perversidad que supone que la miseria se encuentre instalada dentro de la opulencia y el despilfarro. La perplejidad que este entorno ha provocado orientó la mirada de pensadores, teóricos y activistas hacia aquellos trabajos y teorías que, surgidas varias décadas atrás también en entornos de crisis, buscaron salidas nuevas; unas veces por medio del reformismo, otras con estrategias radicales. Estas últimas dejaron profunda huella en nuestra contemporaneidad. Es el caso de las teorizaciones y proposiciones de Iván Illich — analizadas en este trabajo- a cuya obra se ha recurrido en la afanosa búsqueda de respuestas a la actual crisis.

Por otra parte, en el ámbito más estrictamente pedagógico, en el momento actual algunos historiadores de la educación han vuelto a plantear la pedagogización de la sociedad como un problema y, en consecuencia, como tema de reflexión. Esta - dice el profesor Igelmo- «desde una perspectiva 
más contemporánea se presenta como un fenómeno que rebasa el marco de las instituciones educativas y se instala en su periferia» (p. 12). Se trata de la «tendencia a resolver problemas sociales, económicos, políticos o incluso psicológicos aplicando no solo ecuaciones educativas, sino también métodos pedagógicos» (p. 12). Una mirada analítica sobre este fenómeno ha permitido no solo constatar un crecimiento cuantitativo acelerado, sino señalar - como observa el autor - «las connotaciones nocivas que conlleva su expansión» (p. 13). Así, se ha considerado que las consecuencias de «más entrenamiento, tratamiento, educación y cuidado pedagógico» suponen «una creciente dependencia, tutela, cuidados maternales, condescendencia o infantilización». Este mundo pedagogizado mantendría, según sus críticos, «una gran distancia con los proyectos que buscan desarrollar una mayor autonomía, libertad e independencia» (pp. 13-14).

Parece un buen momento, pues, para la publicación de un trabajo que retoma y analiza la obra de quien también publicó sus libros en tiempos de crisis y realizó propuestas radicales. Coincidieron sus publicaciones - y no es casualidad - con el desencanto que, en los países industrializados, se hizo patente con respecto a la educación y con el declinar del optimismo en relación a la institución escolar como gestora de igualdad; se comprobaba, por entonces, que los esfuerzos por la democratización de la enseñanza realizados no habían dado los resultados esperados. La concepción de la educación como fuente de progreso declinó, lo que vino a generar una visión de crisis autoalimentada, por otra parte, por una literatura muy crítica y extensa sobre las debilidades de la institución escolar señalando, además, la necesidad de cambios. En la región latinoamericana se vivió, entre 1960 y 1980, un verdadero "cambio de frentes», como señaló el profesor R. Nassif. Este periodo estuvo caracterizado por modificaciones importantes en la fundamentación de la educación, y por una creciente actitud crítica que se dirigió no solo a la educación, sino a la sociedad latinoamericana en su totalidad, en la que se acentuaban aceleradamente los problemas y las contradicciones originadas en el contexto político, social e ideológico. Las propuestas de solución a los problemas de la educación y de la escuela tomaron fundamentalmente dos caminos. Por un lado, las denominadas "pedagogías desarrollistas» ofrecieron sus propuestas desde la idea de que los países receptores eran «subdesarrollados»; en ellos, para lograr el «desarrollo», había que dar prioridad en el currículo a todos aquellos contenidos que tendieran al cre- 
cimiento económico. De ahí que propiciaran reformas dirigidas a lograr el adiestramiento de mano de obra cualificada. Frente a esta tendencia pedagógica se sitúan las denominadas «pedagogías de la liberación» que interpretan la realidad económica en otros términos; así, el atraso económico tiene su origen en la dependencia que históricamente ha sufrido la región. Sus propuestas educativas son, en consecuencia, muy diferentes a las desarrollistas. Las pedagogías de la liberación se manifestaron de forma diversa, pero, a pesar de sus variadas expresiones, se reconocen en la común denuncia de los factores de alienación provenientes del contexto económico y político, en las propuestas de transformaciones de la realidad y en la aceptación de la politicidad del hecho educativo.

Es en este contexto histórico de contradicciones, meditaciones, resistencias y nuevas búsquedas en el que da comienzo y se desarrolla la actividad teórica y práctica de Illich en América Latina. En este escenario, la obra de Illich ocupó un lugar destacado con la propuesta de una solución radical a los males de la escuela de aquel momento. Es importante recordar que la crítica de Illich no se limitó a la escuela, sino que abarcó globalmente a la sociedad industrial avanzada, como se decía en aquel momento, fuese capitalista o socialista, y a sus instituciones.

El trabajo del profesor Igelmo comienza con una «Introducción» en la que se plantea una cuestión que orienta el posterior desarrollo del texto. Se pregunta acerca del interés que pueda tener la obra de Illich y su crítica a las instituciones escolares en el siglo XXI, teniendo en cuenta que él mismo se distanció del estilo y del poco atino teórico de La sociedad desescolarizada. La solución a dicha pregunta inicial se expone en el primer capítulo del libro. Allí se señala la necesidad de considerar que el trabajo del pensador de Cuernavaca es más amplio que sus libros y artículos, y así hay que considerarlo para poder aquilatar, en su verdadera dimensión, su obra. Observa, el profesor Igelmo, que la praxis fue un elemento relevante en la biografía personal e intelectual de Illich, y que hay que tenerla en cuenta para acercarse con acierto a su pensamiento. En este sentido, considera de gran importancia la experiencia que desarrolló en América Latina como coordinador en diferentes centros de formación y difusión del pensamiento (Puerto Rico, México y Brasil); la práctica fue, pues, un aspecto consustancial al desarrollo de las ideas illichianas. Se describe, en este capítulo, el itinerario seguido por Illich y 
la actividad realizada en los primeros centros de formación, así como la creación del CIDOC, centro de documentación que constituyó una base fundamental para la investigación del momento y futura. Fue un espacio de convivencia y de aprendizaje en el que, poco a poco, se construyó un pensamiento crítico latinoamericano y mundial, expuesto en $\mathrm{La} C \mathrm{Convi}$ vencialidad, obra en la que se analiza el acontecer del mundo en aquella época - finales de los años sesenta y principios de los setenta-. Para el profesor Igelmo, la actividad de Illich en estos centros de formación fue básica para comenzar a idear las alternativas a las instituciones escolares que fueron expuestas en La sociedad desescolarizada.

En el capítulo segundo, el autor analiza las ideas y las tesis desarrolladas por Illich en La Sociedad desescolarizada, así como en una serie de publicaciones previas a la edición de dicha obra. Esta tarea de búsqueda le permite reconstruir la genealogía de este polémico escrito al constatar que los capítulos que lo componen habían ido apareciendo previamente, en distintos formatos, antes de su publicación, en 1971, en Nueva York. Demuestra cómo la crítica de Illich en esta obra se orienta no solo a un cuestionamiento radical del trabajo que se desarrolla en el interior del espacio escolar, sino que también juzga severamente el rol que las instituciones educativas desempeñan en el mantenimiento del mito del progreso, sostenido por el modelo de desarrollo industrial avanzado. Es en esta obra donde propone un conjunto de posibles alternativas para la acción partiendo de la idea de que dichas alternativas a los grandes sistemas escolares deben buscar su centro de desarrollo en la propia experiencia humana.

A partir del estudio de los textos críticos con las instituciones escolares publicados en los años sesenta y setenta, el profesor Igelmo marca dos líneas de análisis que le permiten concluir que, en primer lugar, La sociedad desescolarizada "difícilmente puede ser concebida como un trabajo de referencia entre quienes buscan articular en el siglo XXI una crítica histórica o pedagógica a las instituciones educativas modernas» (p. 20); en segundo término, afirma que los textos publicados por Illich «constituyen una referencia para orientar la acción política y social en oposición a la pedagogización» (p. 20).

En el último capítulo, el autor advierte acerca del error que supone situar La sociedad desescolarizada como principio y fin del pensamiento 
de Illich. Estudia las hipótesis sobre la desescolarización en el contexto en que esta obra apareció, así como el discurso educativo moderno que se elaboró a partir de una nueva lectura de esta obra, en los años ochenta y noventa, buscando alternativas pedagógicas. En este último capítulo, el autor señala que el interés que ha vuelto a despertar la obra de Illich no suele tener en cuenta la complejidad de su trayectoria intelectual - ya analizada en el primer capítulo- y esta visión, por ser parcial, afirma el profesor Igelmo, conduce a errores. Así, por ejemplo, hay defensores de la educación de sus hijos en el hogar que basan sus argumentos en las tesis de Illich pasando por alto sus reflexiones posteriores cuando habló de la dependencia que generaba la expansión de la educación dentro de la familia. Analiza también la pedagogización actual desde la perspectiva que ofrecen los escritos de Illich. Un apéndice con interesantes reflexiones acerca del interés — «interés fluctuante» (p. 191)—que ha despertado la obra de Illich en las últimas décadas finaliza el libro.

La obra de Illich, especialmente por las propuestas que expone en La sociedad desescolarizada, fue muy criticada en su momento. Se dijo que estaba poco fundamentada, que era fantasiosa e idealista, que su arrolladora fuerza crítica no estuvo a la altura de sus débiles propuestas prácticas. Así, R. Nassif, uno de los críticos más destacados de la obra de Illich, afirmó que la desescolarización «no pasa de ser el fruto de una especulación intelectual que no ha producido una práctica real ni la ha encontrado para probar plenamente sus hipótesis». ${ }^{1}$ Pero más allá de las críticas que vieron inviable su idea de un mundo sin escuelas no puede olvidarse que su proposición de las redes de aprendizaje se concretó y se desarrolló efectivamente fuera de la educación formal. Esta forma de enseñanza y aprendizaje ha ido arraigando, y hoy son muchas y cada vez más las ofertas educativas que se llevan a cabo fuera de la escuela.

Las críticas no deben ocultar el hecho de que este pensador ha realizado aportaciones importantes al pensamiento pedagógico. La controversia que suscitó su obra constituyó un elemento básico para la crítica y

\footnotetext{
${ }^{1}$ Ricardo Nassif, El sistema educativo en América Latina (Buenos Aires: UNESCO/CEPAL/PNUD/ Kapelusz, 1984), 65. Conviene recordar que la crítica de Nassif se hizo partiendo de la realidad educativa latinoamericana de aquel momento. Los altos índices de analfabetismo, de abandono escolar, de repetición y la existencia de millones de niños en edad escolar sin escolarizar delimitaban el perfil de la educación latinoamericana. En esas circunstancias, las tesis de Illich expuestas en La sociedad desescolarizada se percibieron ajenas a aquella realidad.
} 
la reflexión pedagógicas, una perspectiva indispensable para el desarrollo y la evolución de los procesos educativos. Sus textos, más que una propuesta alternativa a la escuela, constituyeron y constituyen un estímulo, un llamamiento a la imaginación pedagógica. Por otra parte, es muy difícil no aceptar el planteamiento de Illich sobre la escuela sustentada por las sociedades industriales avanzadas, y su carácter reproductor de las debilidades y perversiones del capitalismo industrial. Es difícil también dejar a un lado su idea de recrear la sociedad para que el individuo adquiera la autonomía y creatividad a la que como ser humano está llamado. De ahí que tuviera muchos partidarios que apostaron por sus ideas para fundamentar la necesidad de construir un mundo más justo.

Mucho tiempo después de la eclosión de las hipótesis de Illich, sus ideas afloran nuevamente formando parte de nuevas teorías que rechazan la dinámica funesta del capitalismo actual que actúa debilitando los lazos de solidaridad y, en consecuencia, erosionando la cohesión social. Es el caso, por ejemplo, de la teoría del «decrecimiento» que en su origen reúne distintas corrientes de pensamiento representadas por grupos que han venido luchando, durante las últimas décadas, contra el crecimiento sin límites. En esta teoría, propuesta como alternativa, están presentes las hipótesis de Illich presentadas por un grupo de sus seguidores, entre los que destaca Serge Latouche, considerado el padre de este movimiento y, a la vez, alumno de Illich.

Esta nueva lectura de la obra de Iván Illich, que nos propone el profesor Igelmo, está unida, pues, a un contexto de rehabilitación del pensamiento illichiano, en el que se ha retomado el debate en torno a su obra que vuelve a actualizarse. El libro del profesor Igelmo es una buena guía, muy bien documentada, para quien quiera acercarse nuevamente, o por primera vez, a este original y polémico pensador.

Miryam Carreño Rivero Universidad Complutense de Madrid miryamcrivero@gmail.com 